\title{
Desarrollo morfológico y conductual de pollos del aguilucho chico Buteo albigula (Aves: Accipitridae) en el noroeste de la Patagonia argentina
}

\author{
Morphologic and behavioral development of white-throated hawk Buteo albigula \\ (Aves: Accipitridae) nestlings in northwestern Argentine Patagonia

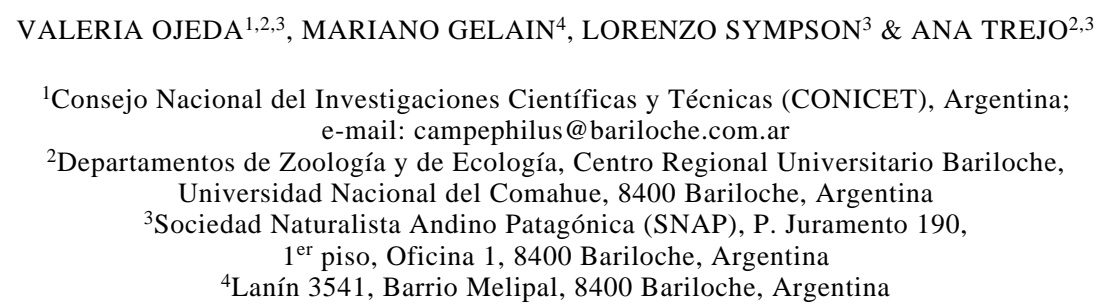

RESUMEN

El desarrollo morfológico y conductual del aguilucho chico (Buteo albigula) en el nido fue estudiado en cercanías de San Carlos de Bariloche, al noroeste de la Patagonia argentina. El estudio se basó en el seguimiento de dos pollos producidos en diferentes nidos en la temporada reproductiva 2001-2002, y se lo complementó con datos tomados ad libitum en otros nidos, esa misma y anteriores temporadas. Los pollos permanecieron en el nido por aproximadamente seis semanas. Durante su desarrollo morfológico se evidenciaron diversos cambios; notablemente, la aparición secuencial de dos plumones natales, que solo fue observada entre especies de Buteo neotropicales, para B. brachyurus. La conducta de los pollos se encuadró en los patrones comunes para otras especies de Buteo de tamaño corporal similar. Además, se caracterizó, por primera vez para la Argentina, un juvenil (muerto accidentalmente), y se tomaron medidas y peso (por primera vez para B. albigula) de dos adultos anillados cerca de sus nidos. El juvenil fue relativamente similar al descrito en Chile. Los adultos, que serían los primeros aguiluchos chicos en ser anillados, se suman a los escasos ejemplares medidos en el área reproductiva conocida de la especie.

Palabras clave: aguilucho chico, Buteo albigula, desarrollo del pollo, descripción del juvenil, morfometría y peso de adultos.

\begin{abstract}
The morphological and behavioral development of white-throated hawk (Buteo albigula) nestlings was studied near San Carlos de Bariloche, in northwestern Argentine Patagonia. The study was mainly based on the monitoring of two nestlings produced at different nests during 2001-2002 breeding season, and was reinforced with data obtained at libitum in other nests the same and previous breeding seasons. The nestling period extended for approximately 6 weeks. Morphological development was characterized by several changing features, but the most surprising was the development of first and second natal down, which was only known, among Neotropical Buteo species, for B. brachyurus. Nestling behavioral development matched the general patterns observed for Buteo spp. of comparable body size. Besides, a juvenile (dead accidentally) was characterized for the first time in Argentina, and two adults, banded near their nests, were measured and weighed (first data for B. albigula). The juvenile was relatively similar to that described in Chile. The adults, which would be the first white-throated hawks ever banded, add to the few specimens measured in the breeding range known for the species.
\end{abstract}

Key words: adult weight and measurements, Buteo albigula, juvenile description, nestling development, white-throated hawk. 


\section{INTRODUCCIÓN}

Para muchas especies de Falconiformes se carece de descripciones morfológicas y conductuales de las primeras fases del desarrollo, especialmente aquellas que transcurren en el nido. Una de estas especies es el aguilucho chico (Buteo albigula Philippi 1899), de estatus taxonómico y migratorio históricamente confusos, y cuya historia natural es casi desconocida en toda su distribución (Brown \& Amadon 1968, del Hoyo et al. 1994, Bierregaard 1998, Ferguson-Lees \& Christie 2001), que abarca ambos lados de la cordillera de los Andes, desde el noroeste de Venezuela hasta el sur de Argentina y Chile. Solo en los últimos dos países se ha registrado la reproducción de esta especie, y recientemente se han dado a conocer algunos datos acerca de su biología (Casas \& Gelain 1995, Pavez 2000, Gelain et al. 2001, Figueroa et al. 2001, Trejo et al. 2001).

A la fecha existen escasas descripciones morfológicas publicadas del aguilucho chico, incluso de ejemplares adultos, y no existen datos de peso para esta especie (Ferguson-Lees \& Christie 2001). Además, la mayoría de las fuentes primarias con información original hace referencia a ejemplares capturados en el norte de Sudamérica, fuera del rango de reproducción conocido para esta especie. Allí, Stresemann (1959) redeterminó y caracterizó siete especímenes provenientes de Perú, Venezuela, Ecuador y Colombia (previamente determinados como B. brachyurus), y revisó los caracteres de ambas especies. Lehmann \& Haffer (1960) describieron y midieron nueve ejemplares (cinco adultos y cuatro juveniles) recolectados en Colombia y Ecuador, resaltando las características diagnósticas de B. albigula, y las que permiten diferenciarlo de B. brachyurus. Rand (1960) caracterizó cinco ejemplares (tres adultos y dos inmaduros) provenientes de Colombia, Ecuador y Perú.

Los ejemplares descritos en el extremo sur de la distribución del aguilucho chico, donde se reproduce con seguridad, son aún más escasos. En Chile, Goodall et al. (1951) dieron medidas de seis especímenes, cinco adultos provenientes de zonas boscosas del centro y centro sur del país (entre los que se encuentra el tipo de la especie), y describieron con cierto detalle un ejemplar inmaduro recolectado en junio en el norte de Chile, cerca de Vallenar. Si bien Johnson (1965) comentó que en Chile en años subsiguientes "varios otros ejemplares han salido a la luz", el autor no reportó medidas o descripciones de los mismos. En Argentina, los ejemplares descritos son cinco adultos capturados en el noroeste patagónico. De estos, dos machos y una hembra fueron descritos y medidos por Navas \& Manghi (1991) y otros dos ejemplares (uno de cada sexo) por Casas \& Gelain (1995).

En este trabajo presentamos una descripción morfológica y conductual de las primeras fases del desarrollo de B. albigula, basada principalmente en datos tomados en dos nidos estudiados durante la temporada reproductiva 2001-2002 en el noroeste de la Patagonia argentina. Dicha descripción se complementa con información obtenida ad libitum en otros nidos hallados desde 1998. Además, dado que no existen ejemplares juveniles descritos para la Argentina, caracterizamos un juvenil (de edad conocida) muerto de manera accidental. Aportamos también datos morfométricos y el peso de dos adultos capturados y anillados en inmediaciones de sus nidos.

\section{MATERIALES Y MÉTODOS}

El estudio se realizó en los alrededores de San Carlos de Bariloche $\left(41^{\circ} 08^{\prime} \mathrm{S}, 71^{\circ} 12^{\prime} \mathrm{O}\right)$, al noroeste de la Patagonia argentina. La zona es típicamente cordillerana, con lagos, valles glaciales y montañas parcialmente cubiertas por bosques de Nothofagus spp. y, en menor proporción, ciprés de la cordillera (Austrocedrus chilensis). La elevación del área oscila entre los 400 y los $3.478 \mathrm{~m}$ de altitud, y el clima es muy estacional. La temperatura media anual es de 8 ${ }^{\circ} \mathrm{C}$ y la precipitación anual oscila entre 500 y $2.000 \mathrm{~mm}$, estando principalmente concentrada en el invierno (Paruelo et al. 1998).

Desde 1998 hemos estudiado diferentes aspectos de la biología del aguilucho chico en bosques cercanos a San Carlos de Bariloche, donde localizamos cuatro territorios reproductivos (descritos por Gelain et al. 2001) que fueron reutilizados en años sucesivos, lo que nos permitió tomar datos ad libitum, y programar un estudio intensivo de la biología reproductiva de esta especie. El mismo fue llevado a cabo entre noviembre de 2001 y enero de 2002 en bosques puros y maduros de lenga ( $N$. pumilio) del Valle del Arroyo Challhuaco, situado $15 \mathrm{~km}$ al SE de San Carlos de Bariloche. Se estudiaron dos nidos activos (N1 y N2) que fueron detectados durante la incubación, momento a partir del que se visitaron semanalmente hasta su abandono por los pollos. En cada visita se registró toda actividad observada en los nidos durante la jornada completa (del amanecer al anochecer, unas $16 \mathrm{~h}$ ). Las observaciones fueron efectuadas desde sitios del bosque con buena visibilidad de los nidos, desde escondites ubicados a distancias de entre 20 y $25 \mathrm{~m}$, y utilizando binoculares y telescopios. Se obtuvo así 
una descripción secuencial del aspecto de los pollos a lo largo del período de nidificación, con sus correspondientes correlatos conductuales. Un tercer juvenil, nacido esa misma temporada en el área, fue muerto accidentalmente al ser atropellado por una cabina de teleférico, luego de lo cual fue recolectado aún fresco.

Dado que $B$. albigula no manifiesta dimorfismo sexual obvio (Ferguson-Lees \& Christie 2001), capturamos un macho y una hembra adultos, uno de cada nido monitoreado. Para ello se pusieron a prueba dos técnicas diferentes (Bloom 1987), por ser estas las primeras capturas vivas conocidas para la especie. En un caso se utilizó una trampa Bal-chatri (jaula conteniendo un roedor vivo, y múltiples lazos de alambre en su exterior en los que el ave queda enganchada), colocada a pocos metros del nido, y en el otro, la técnica Dho-gaza (red de neblina interpuesta entre el nido y un búho embalsamado como señuelo). Los individuos capturados fueron marcados con anillos metálicos.

Para pesar las aves se utilizó una pesola de $1.000 \mathrm{~g}$ (sensibilidad $10 \mathrm{~g}$ ), y para medirlas, regla o calibre manual, según la dimensión considerada. Las mediciones se efectuaron según Baldwin et al. (1931). Para la descripción de ejemplares se utilizaron las cartas de colores provistas por Canevari et al. (1991).

\section{RESULTADOS}

\section{Desarrollo del pollo del aguilucho chico}

Un solo pollo fue producido en cada nido (N1 y N2), con una diferencia de eclosión de 3-5 días entre ambos. Su permanencia en el nido fue de alrededor de seis semanas. Basándonos principalmente en ellos, y en otros cinco nidos observados ad libitum, podemos resumir los principales hitos en el desarrollo morfológico del pollo del aguilucho chico. Este permaneció totalmente blanco (excepto perioculares negros) en su plumón natal durante casi toda su primera semana de vida. Más tarde mudó hacia otro plumón blanco grisáceo a gris perla en las partes dorsales, mientras que las partes ventrales se tornaron blanco sucio, permaneciendo blanco inmaculado solo en la garganta. Alrededor de los 10 días comenzaron a evidenciarse los cañones de las plumas del vuelo sobre alas y cola, y rondando los 15 días, comenzaron a aparecer las estrías y pintas pardo oscuras características de la especie sobre el pecho, abdomen y flancos. En las partes ventrales inferiores, las estrías se unían los flancos del mismo color, haciéndolos parecer más sólidos. Entretanto, el negro del periocular se hizo más evidente y fue avanzando hacia la cera, llegando a formar un antifaz alrededor del día 20. En este momento, las rémiges mostraban cierto desarrollo, formando un notable borde pardo oscuro a negruzco en el ala. La transformación de las partes dorsales cenicientas hacia la coloración pardo oscura a pardo negruzca con que el pollo abandonó el nido, comenzó al promediar el día 20, evidenciándose en forma de manchas pardo oscuras sobre el fondo grisáceo, que representaban el crecimiento de plumas cobertoras aisladas. El aspecto "escamado" característico del dorso de B. albigula (debido al borde rufo de las cobertoras) fue notado entre los días 25 y 30, cuando las partes dorsales ya estaban completamente emplumadas. La cabeza, en cambio, aún permanecía básicamente grisácea con estrías pardo oscuro y blancas. Alrededor de los 30 días el antifaz se hizo menos perceptible, ya que la cabeza tomó colores más oscuros. A partir de ese momento el desarrollo general del plumaje fue muy notorio, y el pecho, abdomen y flancos, que ya estaban fuertemente estriados de pardo oscuro, comenzaron a teñirse de ante canela a rufo leonado (según el ejemplar y la región ventral del cuerpo), mientras que permanecía blanca la garganta (apenas lavada de ante) y blanco sucio el vientre. Los calzones se tiñeron muy intensamente de leonado. Con esta coloración ventral el pollo dejó el nido, conservando blanca solo una pequeña zona, en la barba, que no era notoria. Hacia el día 32-33, los lados del cuello se tornaron pardo leonado, y la cabeza veteada de gris, pardo oscuro, y blanco, con el antifaz poco visible. Por último (entre los $35 \mathrm{y}$ 40 días), la cabeza se tornó netamente pardo oscura, aunque dorsalmente muy jaspeada de blanco y rufo o leonado, y a veces, aun el gris del segundo plumón. El antifaz era casi imperceptible. La coloración del pico (gris negruzco), la cera (amarillo limón a amarillo claro), el tarso (amarillo limón a amarillo claro), la garra (negra) y la pupila (pardo claro) se sostuvo esencialmente constante durante el desarrollo.

El pollo del aguilucho chico mantuvo una elevada tasa de actividad. Desde los primeros días de vida se desplazaba por el nido, defecaba hacia fuera de este, y era capaz de ensayar prácticas de alimentación (por ejemplo, picotear presas o golpearlas), aunque fue alimentado por adultos con trozos pequeños de carne roja, sin faneras o partes duras, durante la primera semana de vida. También en ese período permaneció la mayor parte del día bajo el cuerpo de la hembra. Los avances conductuales observados a partir de la primera semana se resumen básicamente en: (1) un aumento gradual en la coordinación motriz del pollo, que a la semana ya recorría 
completamente el nido, a los 10 días se mantenía erguido, a los 15 días batía sus alas, y a los 30 días salía a caminar y aletear fuera del nido, y saltaba y corría dentro del mismo, (2) la adquisición gradual de la capacidad de tragar trozos de alimento grandes y sin limpiar de partes duras, lo cual lo llevó a alimentarse solo alrededor del día 20, (3) la de acicalarse, notada repentinamente el día 21, (4) la de buscar el mejor microclima disponible en el nido, registrada el día 15, (5) la de responder a estímulos en el entorno del nido, entre los 15 y 20 días, y (6) la de picotear ramas, observada hacia el día 15. En síntesis, a partir de la tercera semana de vida (entre 15 y 20 días), los pollos adquirieron un conjunto de nuevos comportamientos que se tradujeron en un notorio cambio de actitud.

El abandono del árbol-nido tomó alrededor de una semana, la sexta desde el nacimiento. Durante este proceso, que fue observado en N1 y $\mathrm{N} 2$ y en otros 5 nidos, el pollo recorría diferentes ramas del árbol-nido, ya sea caminando o dando pequeños saltos acompañados de aleteos. Durante los primeros días en que el pollo se dedicaba a recorrer el árbol-nido, los adultos abandonaban el sitio por largas horas, y pocas veces al día alimentaban al pollo. El día de los primeros vuelos significativos (fuera del árbol-nido), los adultos ya no visitaban el nido y depositaban las presas en perchas cercanas. Durante estas interacciones las vocalizaciones de adultos y pollo eran frecuentes. A los dos o tres días de volar, el pollo era capaz de seguir a sus padres fuera de las inmediaciones del nido, y nunca observamos juveniles o adultos utilizando el nido en días posteriores, al menos durante el día.

Descripción del juvenil y medidas de los adultos

Las medidas del juvenil accidentado (un macho de dos meses y medio de vida) se detallan en la Tabla 1, junto con las de los adultos captura- dos. El juvenil, cuyo plumaje no evidenciaba desgaste, se describe a continuación. Cabeza: por encima, pardo negruzco, jaspeado de blanco y ante canela en corona, nuca y lados. Región auricular pardo negruzco mezclado con rufo leonado. Frente y mejillas ante. Laterales de la base del pico crema anteado. Barba y párpados inferiores blancos apenas lavados de crema o marfil. Fino anillo alrededor del ojo pardo negruzco muy oscuro, color que se continuaba en lorales. Cera amarillo verdoso. Iris pardo. Partes dorsales: mayormente pardo negruzco, dado por plumas muy oscuras de base blanca (no visible), y margen rufo leonado. Estos márgenes eran más notorios en escapulares y cobertoras superiores del ala y en la rabadilla, que tomaban un aspecto escamado. Partes ventrales: garganta y cuello ante canela, limitando anteriormente con la barba, más clara. A continuación, pecho, abdomen, flancos e infracaudales ante, jaspeados de pardo oscuro. En el pecho, las estrías eran finas, y se iban ensanchando y concentrando hacia los flancos, por lo que estos parecían más uniformemente oscuros. En el abdomen, estrías anchas y angostas, dispersas. Cola: en vista dorsal, pardo negruzco. Rectrices con barrado no muy notable formado por nueve bandas pardo negruzco (de alrededor de $4 \mathrm{~mm}$ las ocho más proximales, y de $10 \mathrm{~mm}$ la más distal), sobre un fondo pardo grisáceo oscuro en bandas de igual ancho (aproximadamente 6 $\mathrm{mm})$. Margen final de las rectrices $(4,5 \mathrm{~mm})$ crema o marfil. Raquis negro. En vista ventral, la cola era color cuerno y aspecto perlado y se observaba un barrado muy poco notable, de unas 10 bandas que se iban aclarando desde el extremo hacia la base. Raquis blanco. Ala: en vista dorsal, escapulares y cobertoras superiores pardo negruzco con fino reborde rufo leonado. Primarias pardo negruzco (el extremo parecía casi negro), salvo en los dos tercios proximales de la lámina interna, que eran blanquecinos con bandas angostas pardo oscuro que

TABLA 1

Medidas de un juvenil y dos adultos del aguilucho chico Buteo albigula provenientes de San Carlos de Bariloche, Argentina. Peso en g y medidas lineales en $\mathrm{mm}$

Measurements of a juvenile and two adult white-throated Hawks Buteo albigula from San Carlos de Bariloche, Argentina. Weight in $\mathrm{g}$ and other body measurements in $\mathrm{mm}$

\begin{tabular}{lccccc}
\hline Sexo & Peso & Largo total & Cuerda del ala & Cola & Culmen \\
\hline$q$ adulto & 600 & - & 300 & 170 & 183 \\
$\sigma^{\top}$ adulto & 525 & 415 & 305 & 195 & 140 \\
$\sigma^{r}$ juvenil & - & 430 & 289 & 182 & 164 \\
\hline
\end{tabular}


se acentuaban hacia las secundarias. Además, las cuatro primarias más externas tenían una muesca en el tercio distal que acentuaba el oscurecimiento del extremo de las primarias. Secundarias también pardo negruzcas, salvo en los dos tercios proximales de la lámina interna, que eran de fondo gris parduzco claro, y con bandas angostas pardo oscuro. Ventralmente, subalares primarias ante canela finamente estriadas de pardo oscuro. Las subalares secundarias menores mantenían la coloración y el fino estriado de las subalares primarias. Las subalares secundarias medias y mayores mantenían la coloración ante canela, pero el estriado pardo oscuro era más grueso. Primarias blancas en sus dos tercios proximales que, a partir de la cuarta primaria, comenzaban a estar barrados de pardo negruzco, muy notable hacia las secundarias. Extremo de las primarias oscuro, dado por barras pardo negruzco sobre un fondo pardo grisáceo (en las cuatro más externas, el oscurecimiento se daba a partir de la muesca). Secundarias gris claro (con cierto reflejo plateado) con bandas angostas gris oscuro (barrado muy difuso) y ancha banda terminal de igual color. Tibia: leonado con manchas pardas irregulares, anchas y cortas. Las mismas eran producidas por un barrado incompleto y espaciado. Tarso y pie: amarillo-verdoso. Garra: negra.

\section{DISCUSIÓN}

Los principales hitos observados en el desarrollo de los pollos en los nidos monitoreados fueron coincidentes, en general, con lo registrado para pollos de otros nidos, durante esa misma temporada y en años anteriores.

Dada la ausencia de información previa sobre el desarrollo conductual o morfológico del aguilucho chico en el nido, no es sencillo referenciar los resultados de este estudio. Las restantes dos especies de Buteo que habitan los bosques templados de Argentina y Chile, el aguilucho de cola rojiza (B. ventralis) y el aguilucho común ( $B$. polyosoma), son prácticamente desconocidas en los aspectos tratados en este trabajo (e.g., del Hoyo et al. 1994). Solo para el aguilucho común se describe someramente al pollo, del cual se dice que tiene plumón rosa salmón (del Hoyo et al. 1994, aunque ver Pavez 1998), y que permanece en el nido entre 40 y 50 días (Jiménez 1995) o más (del Hoyo et al. 1994), tiempo algo mayor que el observado para B. albigula. Al margen de esta falta de datos, otro aspecto de la comparación con los aguiluchos residentes en los bosques templados australes a tener en cuenta es que ambos son de tamaño corporal mayor que el del aguilucho chico, lo cual podría ser motivo de diferencias en el desarrollo de los pollos, particularmente en lo relativo a los tiempos involucrados.

En una revisión de la información disponible sobre desarrollo de pollos para otros Buteo neotropicales continentales hallamos que tampoco existen demasiados datos sobre esta etapa de la vida para la mayoría de las especies (del Hoyo et al. 1994). Sin embargo, ciertas relaciones interesantes fueron notadas. El aguilucho de cola corta (B. brachyurus) presenta la particularidad de que su pollo pasa por dos plumones antes de adquirir el plumaje juvenil, que son color blanco y gris, tal como observamos en el aguilucho chico. Es más, en ningún otro aguilucho (Buteo spp.) para los que existen descripciones de pichones (unas ocho especies) se registró este pasaje secuencial por dos plumones natales diferentes. Esto reforzaría la proposición, hecha en base a caracteres morfológicos, de que el aguilucho chico y el de cola corta forman una unidad taxonómica, siendo quizás razas de una especie politípica (Rand 1960) o especies de una superespecie (del Hoyo et al. 1994). Con respecto al tiempo de permanencia en el nido, lamentablemente, el mismo no es conocido para B. brachyurus (así como muchos otros aspectos de su desarrollo). Tomando en cuenta el resto de los Buteo continentales neotropicales, podemos decir que el agilucho chico sigue el patrón general (entre 40 y 45 días), si nos restringimos a aquellas especies de tamaño corporal equiparable.

La aparición de las plumas de contorno se dio de manera diferente de la esperada, ya que lo común en las distintas especies de Buteo es observarla en el segundo tercio del período de nidificación (del Hoyo et al. 1994), mientras que en el aguilucho chico se dio antes de finalizado el primero (rondando los 10 días). También la alimentación sin asistencia de los adultos fue precoz en el aguilucho chico respecto de lo conocido para su género, pues se observó al comenzar el segundo tercio del período de nidificación, mientras que generalmente ocurre en el último tercio (del Hoyo et al. 1994). Es común, en varias especies de Buteo, que la tasa de desarrollo se detenga y los pollos incluso pierdan peso debido a una reducción en el flujo de alimento durante los últimos días de permanencia en el nido (del Hoyo et al. 1994). Si bien nosotros no pesamos los pollos (ante el riesgo de que sean abandonados por sus padres), estos no recibieron alimento durante la última semana de observación.

El número promedio de juveniles producidos por nido en especies de Buteo de zonas templa- 
das varía entre 1.5 y 2.6 (del Hoyo et al. 1994). El hecho de que los juveniles de B. albigula deban abandonar el territorio reproductivo a poco de haber volado, debido al carácter migratorio de la especie, puede estar imponiendo restricciones a la cantidad de pollos que es viable criar con éxito cada temporada. En muchos de los accipítridos migradores, la inversión parental en los días y semanas luego de que el pollo ha abandonado el nido continúa siendo muy alta, debido a que estos juveniles deben acelerar su aprendizaje de técnicas de vuelo y cacería, lo que es frecuentemente mediado por los adultos (del Hoyo et al. 1994). Lamentablemente, nuestros datos sobre el comportamiento del juvenil una vez abandonado el nido son escasos, y se remiten a algunas observaciones de vuelos en parejas o de a tres individuos cerca de territorios reproductivos, donde hemos notado que el juvenil (que manifiesta formas de vuelo y voces distinguibles) raramente se distancia de al menos uno de los adultos.

Para cotejar las dimensiones de los adultos medidos en este trabajo, consultamos las medidas de la cuerda del ala, la cola y el culmen en todas las fuentes de las que teníamos conocimiento. El rango de medidas del culmen registrado en la literatura varía desde los 16 (Johnson 1965) a los $22 \mathrm{~mm}$ (Lehmann \& Haffer 1960); nuestras medidas lo amplían, ya que el macho adulto medido en este trabajo tenía un pico notablemente corto, quizás a causa del desgaste. El rango de medidas de la cuerda del ala varía desde los 272 (Rand 1960) a los 327 mm (Lehmann \& Haffer 1960); nuestras medidas están dentro del mismo. El rango de medidas de la longitud de la cola varía desde los 157 (Rand 1960) a los 193 mm (Rand 1960, Johnson 1965), límites que incluyen nuestros datos. En particular, los cinco ejemplares descritos para la Argentina (Navas \& Manghi 1991, Casas \& Gelain 1995) eran notablemente homogéneos en sus dimensiones, y respecto de ellos, nuestros datos incrementan la variación registrada para los tres caracteres. Los ejemplares descritos para Chile eran, en cambio, bastante más variables que los de Argentina, y respecto de ellos solo modificamos el límite inferior del rango de medidas del culmen.

En un análisis conjunto de nuestras medidas y todas las que se citan en la literatura, no pudimos hallar una relación clara entre la variación registrada en las dimensiones de B. albigula y el sexo, la edad o la zona de colección de los ejemplares, excepto por una tendencia de las hembras a tener cola y alas algo más largas que los machos, lo que se evidencia solamente en algunas fuentes, aquellas donde se midieron va- rios ejemplares (Stresemann 1959, Lehmann y Haffer 1960, Goodall et al. 1951). Esto coincidiría con lo esperado para accipítridos, en donde la hembra suele ser de mayor porte (y peso) que el macho de su especie. Sin embargo, cabe destacar que las medidas dadas para B. albigula pueden haber sido tomadas de manera diferente por los distintos autores, razón por la cual no sería del todo confiable un análisis global a partir de ellas. Lamentablemente, no existen datos publicados de peso de los individuos que puedan reforzar el patrón observado en las medidas, aunque, al respecto, cabe destacar que nuestros datos van en el sentido esperado, siendo la hembra algo más pesada que el macho. Pese a que la especie no manifiesta dimorfismo sexual en su plumaje, la leve diferencia registrada en el peso podía ser notada en el campo, con cierto entrenamiento, y si ambos miembros de la pareja eran vistos posados a la vez.

El juvenil que describimos muestra cierta similitud con el descrito por Goodall et al. (1951) para Chile, aunque este último se describió con mucho menor detalle, lo cual impide una buena comparación. Dicho ejemplar presentaba partes ventrales o inferiores color crema y había sido capturado casi en invierno en el norte de Chile (fuera del área reproductiva conocida para la especie), por lo que creemos que podría ser de mayor edad que el juvenil aquí descrito. Nuestro juvenil también se asemeja a algunos de los que se describieron en el norte de Sudamérica (Stresemann 1951, Lehmann \& Haffer 1960, Rand 1960), que presentan entre sí variación en la tonalidad de las partes ventrales, desde "fuertemente teñidas de ocráceo" a "blanco anteado" o "blanco lavado de amarillo-cremoso". Es probable que la coloración ventral intensa (entre ante canela y rufo leonado), observada en los pollos a punto de volar y en el ejemplar accidentado, corresponda a los primeros meses de la vida de esta rapaz, para luego tornarse crema y finalmente blanca, característica de los adultos de $B$. albigula. Se desconoce totalmente el tiempo que tomaría dicha transformación.

A lo largo de cinco primaveras no hemos detectado el arribo a la Patagonia argentina de ejemplares con características físicas netamente juveniles, lo que nos lleva a plantear dos explicaciones posibles: (1) que el juvenil se asemeje físicamente al adulto en menos de un año, independientemente de si se reproducirá o no durante su segunda estancia en los bosques templados australes, o (2) que los juveniles no regresen a dichas áreas reproductivas del sur, sino que permanezcan en latitudes menores durante sus primeros años. La evidencia para clarificar este asunto es al menos escasa, y hasta confusa. 
Pavez (2000) registró, desde dos puntos fijos de observación en Chile central, migraciones masivas de aguiluchos chicos, pero sin hacer distinción entre adultos y juveniles. Sin embargo, este autor publicó también un listado de observaciones ocasionales de la especie entre 1990 y 1998 en diferentes localidades de Chile, donde mencionó un grupo de siete individuos volando en dirección norte-sur en Chacabuco $\left(33^{\circ} 00^{\prime} \mathrm{S}\right.$, $70^{\circ} 40^{\prime} \mathrm{O}$ ) en primavera, que incluía a dos inmaduros. De dicho listado, nos resultó también llamativa la ausencia de juveniles migrando en dirección contraria en otoño en las cuatro instancias registradas por el autor, quien observó un total de 10 individuos (solo adultos).

$\mathrm{Si}$ bien este es un trabajo de características netamente descriptivas, y basado en un número relativamente pequeño de ejemplares, consideramos que la falta de estudios sobre esta especie hace importante el conocimiento de todos los aspectos de su biología a fin de desarrollar futuras líneas de investigación que permitan su conservación en la que parece ser su principal área reproductiva.

\section{AGRADECIMIENTOS}

Marc Bechard, Graham Frye, Kuky y Carlos Kovacs, Patricio Wallace, Kamille Rogers, Roberto Orduna y Agustín Lanusse contribuyeron a realizar las capturas de individuos y a su caracterización. Susana Seijas, Juan Karlanian, Martín Costa y Lorien Zamorano colaboraron en distintas tareas en terreno y laboratorio. François Vuilleumier aportó bibliografía relevante. Empleados del Teleférico Cerro Otto proporcionaron información muy valiosa para este estudio. Personal del Refugio Neumeyer nos facilitó el uso de sus instalaciones. Los anillamientos se realizaron bajo permiso de la Administración de Parques Nacionales (APN) de Argentina, con anillos del Instituto Miguel Lillo (Tucumán, Argentina).

\section{LITERATURA CITADA}

BALDWIN SP, HC OBERHOLSER \& LG WORLEY (1931) Measurements of birds. Scientific Publications of the Cleveland Museum of Natural History (USA) 2: 1-165.

BIERREGAARD MJ (1998) Conservation status of birds of prey in the South American tropics. Journal of Raptor Research 32: 19-27.

BLOOM PH (1987) Capturing and handling raptors. En: Giron-Pendleton BA, BA Millsap, KW Cline \& DM
Bird (eds) Raptor management techniques manual: 99-123. National Wildlife Federation, Washington, District of Columbia, U.S.A.

BROWN L \& D AMADON (1968) Eagles, hawks, and falcons of the world. Country Life Books, London, United Kingdom. 759 pp.

CANEVARI MP, P CANEVARI, GR CARRIZO, G HARRIS, J RODRÍGUEZ MATA \& RJ STRANECK (1991) Nueva guía de las aves argentinas. Tomo I. Fundación Acindar, Buenos Aires, Argentina. $411 \mathrm{pp}$.

CASAS AE \& M GELAIN (1995) Nuevos datos acerca del estatus del aguilucho andino Buteo albigula en la Patagonia argentina. Hornero (Argentina) 14: 40-42.

DEL HOYO J, A ELLIOTT \& J SARGATAL (eds) (1994) Handbook of the birds of the world. Volume 2. New World vultures to guineafowl. Lynx Edicions, Barcelona, Spain. 638 pp.

FERGUSON-LEES J \& DA CHRISTIE (2001) Raptors of the world. Cristopher Helm, London, United Kingdom. 992 pp.

FIGUEROA RA, S CORALES STAPPUNG \& R LÓPEZ RÜBKE (2001) Records of the white-throated hawk (Buteo albigula) and notes on its hunting methods and movements, in the Andes of central-southern Chile. International Hawkwatcher (USA) 4: 3-9.

GELAIN M, V OJEDA, A TREJO, L SYMPSON, G AMICO \& R VIDAL-RUSSELL (2001) Nuevos registros del aguilucho andino (Buteo albigula) en la Patagonia argentina. Hornero (Argentina) 16: 85-88.

GOODALL JD, AW JOHNSON \& RA PHILIPPI (1951) Las aves de Chile, su conocimiento y sus costumbres. Volumen II. Platt Establecimientos Gráficos, Buenos Aires, Argentina. 442 pp.

JIMÉNEZ JE (1995) Historia natural del aguilucho Buteo polyosoma: una revisión. Hornero (Argentina) 14: 1-9.

JOHNSON AW (1965) The birds of Chile and adjacent regions of Argentina, Bolivia and Peru. Volume 1. Platt Establecimientos Gráficos, Buenos Aires, Argentina. 398 pp.

LEHMANN FC \& J HAFFER (1960) Notas sobre Buteo albigula Philippi. Novedades Colombianas (Colombia) $1: 242-255$.

NAVAS JR \& MS MANGHI (1991) Notas sobre Buteo ventralis y Buteo albigula en la Patagonia argentina (Aves, Accipitridae). Revista del Museo Argentino de Ciencias Naturales Bernardino Rivadavia, Zoología (Argentina) 15: 87-94.

PARUELO JM, A BELTRÁN, E JOBBÁGY, OE SALA \& RA GOLLUSCIO (1998) The climate of Patagonia: general patterns and controls on biotic processes. Ecología Austral (Argentina) 8: 85-101.

PAVEZ E (1998) Observaciones sobre el patrón de coloración en machos y hembras de aguilucho (Buteo polyosoma, Quoy y Gaimard, 1824). Boletín Chileno de Ornitología (Chile) 5: 21-23.

PAVEZ E (2000) Migratory movements of the whitethroated hawk (Buteo albigula) in Chile. Journal of Raptor Research 34: 143-147.

RAND AL (1960) Races of the short-tailed hawk, Buteo brachyurus. Auk 77: 448-459

STRESEMANN E (1959) Buteo albigula Philippi, ein in Südamerika weit verbreiteter Bussard. Journal für Ornithologie 100: 337-340.

TREJO A, V OJEDA \& L SYMPSON (2001) First nest records of the white-throated hawk (Buteo albigula) in Argentina. Journal of Raptor Research 35: $169-170$ 